\title{
Positive solutions and eigenvalue intervals for a second order $p$-Laplacian discrete system
}

Jin Zhao ${ }^{1 *}$ (D)

${ }^{*}$ Correspondence:

jchuphd@126.com

${ }^{1}$ College of Science, Hohai

University, Nanjing, China (c) The Author(s) 2018. This article is distributed under the terms of the Creative Commons Attribution 4.0 International License (http://creativecommons.org/licenses/by/4.0/), which permits unrestricted use, distribution, and reproduction in any medium, provided you give appropriate credit to the original author(s) and the source, provide a link to the Creative Commons license, and indicate if changes were made.

\section{Introduction}

In this paper, we investigate the existence of at least one positive solution for the second order $p$-Laplacian discrete boundary value system

$$
\left\{\begin{array}{l}
\Delta\left(\phi\left(\Delta u_{i}(k-1)\right)\right)+f^{i}(k, u(k))=0, \quad k \in N, \\
u(0)=0, \quad u(T+1)=0, \quad i=1,2, \ldots, n,
\end{array}\right.
$$

where $u=\left(u_{1}, u_{2}, \ldots, u_{n}\right), \phi(s)=|s|^{p-2} s(p>1), N=\{1,2, \ldots, T\}, T \geq 1$ is a fixed positive integer and $\Delta u(k)=u(k+1)-u(k)$ is the forward difference operator.

The discrete boundary value problems arise in different fields of research. For instance, they are widely used in discrete optimization, computer science, population genetics, and so on. Therefore, different types of discrete boundary value problems have been studied in the past three decades, here we refer the reader to $[1,2,4,5,7,8,15,19]$. Among those, the existence of positive solutions for the discrete $p$-Laplacian system has attracted special attention $[4,7,19]$. Some classical and well-known tools, such as the variational methods $[3,17]$, the approach of upper and lower solutions $[10,11]$, and some fixed point theorems $[5,9]$, have been widely used.

This paper is mainly motivated by previous paper [6], in which Chu et al. [5, 6] stated and showed that, under adequate assumptions on the nonlinear term $f$, the discrete boundary value problems have at least one positive solution. In this paper, we will choose another adequate cone to prove the main results, which will improve and generalize those results obtained in [6].

\begin{abstract}
order $p$-Laplacian discrete system. As applications, we characterize the eigenvalue intervals for one typical $n$-dimensional system. The proof is based on a well-known
\end{abstract}

MSC: Primary 34B16; secondary 39A10

Keywords: Positive solution; Discrete system; $p$-Laplacian; n-dimensional;

Eigenvalue intervals; Fixed point theorem in cones 
As applications of our new results, we characterize the eigenvalue intervals for the $n$ dimensional system

$$
\left\{\begin{array}{l}
\Delta\left(\phi\left(\Delta u_{i}(k-1)\right)\right)+\lambda h_{i}(k) g^{i}(u(k))=0, \quad k \in N \\
u(0)=0, \quad u(T+1)=0, \quad i=1,2, \ldots, n
\end{array}\right.
$$

where $\lambda>0$ is a positive parameter. We prove that system (2) has at least one positive solution for each $\lambda$ in an explicit eigenvalue interval. Recently, several eigenvalue characterizations for different kinds of boundary value problems have appeared, and we refer the reader to $[5,12,14,16,18]$.

The rest of this paper is organized as follows. In Sect. 2, we introduce some preliminaries which are used to prove the main results. Some results on the existence of at least one positive solution to system (1) are established in Sect. 3. Finally, in Sect. 4 we study the existence of positive solutions for the following discrete system:

$$
\left\{\begin{array}{l}
\Delta\left(\phi\left(\Delta u_{i}(k-1)\right)\right)+h_{i}(k) g^{i}(u(k))=0, \quad k \in N \\
u(0)=0, \quad u(T+1)=0, \quad i=1,2, \ldots, n,
\end{array}\right.
$$

and characterize the eigenvalue intervals for system (2).

\section{Preliminaries}

First let us introduce some notation. For abbreviation, let $\mathbb{R}$ be the set of real numbers, $\mathbb{R}_{+}=[0, \infty), \mathbb{R}_{+}^{n}=\prod_{i=1}^{n} \mathbb{R}_{+}$, and $N^{+}=\{0,1, \ldots, T+1\}$. We will denote by $C\left(N^{+}, \mathbb{R}\right)$ the set of all continuous functions on $N^{+}$(discrete topology) with supremum norm $\|u\|=$ $\max _{k \in N^{+}}|u(k)|$. Then $C\left(N^{+}, \mathbb{R}\right)$ is a Banach space. Given $u, v \in \mathbb{R}^{N}$, the usual inner product formula is denoted by

$$
\langle v, u\rangle=\sum_{i=1}^{N} v_{i} u_{i}
$$

Take $X=C\left(N^{+}, \mathbb{R}\right) \times \cdots \times C\left(N^{+}, \mathbb{R}\right)(n$ copies $)$. For any $u=\left(u_{1}, \ldots, u_{N}\right) \in X$, we define the $v$-norm by

$$
|u|_{v}=\sum_{i=1}^{N} v_{i}\left\|u_{i}\right\|=\sum_{i=1}^{N} v_{i} \cdot \max _{k \in N^{+}}\left|u_{i}(k)\right|,
$$

where $v \in \mathbb{R}_{+}^{N}$ is a fixed vector. It is easy to see that $X$ is a Banach space.

Next we present some well-known tools which will be used throughout the paper.

Lemma 2.1 ([1]) Assume that there exists a function $y \in C\left(N^{+}, \mathbb{R}\right)$ with $y(k) \geq 0$ for all $k \in N^{+}$. If $u \in C\left(N^{+}, \mathbb{R}\right)$ satisfies

$$
\left\{\begin{array}{l}
\Delta^{2} u(k-1)+y(k)=0, \quad k \in N, \\
u(0)=0, \quad u(T+1)=0,
\end{array}\right.
$$


then $u(k) \geq q(k)\|u\|$ for all $k \in N^{+}$, where

$$
q(k)=\min \left\{\frac{T+1-k}{T+1}, \frac{k}{T}\right\}
$$

Remark 2.2 From (4), it is easy to see that $q(k) \geq \frac{1}{T+1}$ for any $k \in N$.

Lemma 2.3 ([9]) If $u \in C\left(N^{+}, \mathbb{R}\right)$ satisfies

$$
\left\{\begin{array}{l}
\Delta(\phi(\Delta u(k-1))) \leq 0, \quad k \in N \\
u(0)=0, \quad u(T+1)=0
\end{array}\right.
$$

then $u(k) \geq q(k)\|u\|$ for $k \in N^{+}$.

Lemma 2.4 ([9]) If $u, y \in C\left(N^{+}, \mathbb{R}\right)$ satisfies

$$
\left\{\begin{array}{l}
\Delta(\phi(\Delta u(k-1))) \leq \Delta(\phi(\Delta y(k-1))), \quad k \in N \\
u(0) \geq y(0), \quad u(T+1) \geq y(T+1),
\end{array}\right.
$$

then $u(k) \geq y(k)$ for $k \in N^{+}$.

Our main tool is a well-known fixed point theorem in cones established in [13], which we recall here for the convenience of the readers. If $D$ is a subset of $X$, we write $D_{K}=D \cap K$ and $\partial_{K} D=(\partial D) \cap K$.

Theorem 2.5 Let $X$ be a Banach space, and let $K$ be a cone in $X$. Assume that $\Omega^{1}, \Omega^{2}$ are open bounded subsets of $X$ with $\Omega_{K}^{1} \neq \emptyset, \bar{\Omega}^{1}{ }_{K} \subset \Omega_{K}^{2}$. Let

$$
T:{\overline{\Omega^{2}}}_{K} \rightarrow K
$$

be a continuous and completely continuous operator such that

(i) $u \neq \lambda$ Tu for $\lambda \in[0,1)$ and $u \in \partial_{K} \Omega^{1}$, and

(ii) there exists $e \in K \backslash\{0\}$ such that $u \neq T u+\lambda$ for all $u \in \partial_{K} \Omega^{2}$ and all $\lambda>0$.

Then $T$ has a fixed point in $\overline{\Omega^{2}}{ }_{K} \backslash \Omega_{K}^{1}$.

\section{Main results}

In this section, we establish an existence result to the discrete $p$-Laplacian system (1). Define a cone $K$ in $X$ by

$$
K=\left\{u=\left(u_{1}, u_{2}, \ldots, u_{n}\right) \in X: \min _{k \in N}|v, u(k)| \geq q(k)|u|_{v}\right\} .
$$

In addition, the operator $S: K \rightarrow X$ is defined by $S=\left(S_{1}, S_{2}, \ldots, S_{n}\right)$ and

$$
S_{i} u(k)= \begin{cases}0, & k=0 \text { or } T+1, \\ \sum_{s=k}^{T} \phi^{-1}\left(\tau_{i}+\sum_{r=1}^{s} f^{i}(r, u(r))\right), & k \in N\end{cases}
$$


where $\tau_{i}$ is a solution of the equation

$$
\phi^{-1}\left(\tau_{i}\right)+\sum_{s=k}^{T} \phi^{-1}\left(\tau_{i}+\sum_{r=1}^{s} f^{i}(r, u(r))\right)=0 .
$$

Lemma 3.1 Suppose that $f^{i}: N^{+} \times \mathbb{R}_{+}^{n} \rightarrow \mathbb{R}_{+}$is continuous for each $i=1,2, \ldots, n$. Then the operator $S$ is continuous and completely continuous. Furthermore, $S: K \rightarrow K$ is well defined.

Proof Since $\phi^{-1}$ is a continuous, strictly increasing function on $\mathbb{R}$ and $\phi^{-1}(\mathbb{R})=\mathbb{R}$, then $\tau_{i}$ is a unique solution of equation (6). Therefore, the operator $S$ is well defined. It follows from [6, Lemma 6.1] that $S: K \rightarrow X$ is continuous and completely continuous. Next, we show that $S$ maps $K$ into $K$.

Since $f^{i}(k, u(k)) \geq 0$ for $k \in N$, by Lemma 2.3, it is clear that

$$
S_{i} u(k) \geq q(k)\left\|S_{i} u\right\| \quad \text { for all } u \in K \text {. }
$$

Therefore

$$
\langle v, S u(k)\rangle \geq q(k)\langle v,\|S u(k)\|\rangle=q(k)|S u(k)|_{v^{\prime}}
$$

which shows that $S(K) \subset K$.

In order to apply Theorem 2.5, we need to select adequate open sets. Define

$$
\Omega^{r}=\left\{u \in X: \min _{k \in N}\langle v, u(k)\rangle<\frac{r}{T+1}\right\} \quad \text { and } \quad B^{r}=\left\{u \in X:|u|_{v}<r\right\} .
$$

Lemma 3.2 $\Omega^{r}, B^{r}$ defined above have the following properties:

(a) $\Omega_{K}^{r}, B_{K}^{r}$ are open relative to $K$.

(b) $B_{K}^{r / T+1} \subset \Omega_{K}^{r} \subset B_{K}^{r}$.

(c) $u \in \partial_{K} \Omega^{r}$ if and only if $u \in K$ and $\min _{k \in N}\langle v, u(k)\rangle=\frac{r}{T+1}$.

(d) If $u \in \partial_{K} \Omega^{r}$, then $\frac{r}{T+1} \leq\langle v, u(k)\rangle \leq r$ for $k \in N$ and $|u|_{\nu} \leq r$.

Proof Since $\min _{k \in N}\langle v, u(k)\rangle$ is continuous (discrete topology), we check at once that (a) is true. (c) is clear since, for each $u \in K$, we have

$$
\min _{k \in N}\langle v, u(k)\rangle \geq q(k)|u|_{v} \geq \frac{1}{T+1}|u|_{v} .
$$

Now let us prove (d). From (c), we have

$$
\frac{1}{T+1}|u|_{v} \leq q(k)|u|_{v} \leq \min _{k \in N}\langle v, u(k)\rangle=\frac{r}{T+1} \quad \text { for } u \in \partial_{K} \Omega^{r} .
$$

Thus $|u|_{v} \leq r$ and $\frac{r}{T+1} \leq\langle v, u(k)\rangle \leq r$ for $k \in N$.

Next we prove (b). Let $u \in B_{K}^{r / T+1}$, then we have $|u|_{v}<\frac{r}{T+1}$, so $\min _{k \in N}\langle v, u(k)\rangle<\frac{r}{T+1}$ and $u \in \Omega_{K}^{r}$. Since $u \in \Omega_{K}^{r}$, for all $k \in N$, we have $\min _{k \in N}\langle v, u(k)\rangle\left\langle\frac{r}{T+1}\right.$ and $\langle v, u(k)\rangle \geq$ $q(k)|u|_{v} \geq \frac{1}{T+1}|u|_{v}$. Hence $|u|_{v}<r$, that is, $\Omega_{K}^{r} \subset B_{K}^{r}$. 
It follows from the above properties that, for each $\delta>r$,

$$
\Omega_{K}^{r}=\left(\Omega^{r} \cap B^{\delta}\right)_{K} \quad \text { and } \quad{\overline{\Omega^{r}}}_{K}=\left(\overline{\Omega^{r} \cap B^{\delta}}\right)_{K} .
$$

Theorem 3.3 Suppose that $f^{i}: N^{+} \times \mathbb{R}_{+}^{n} \rightarrow \mathbb{R}_{+}$is continuous. Moreover, suppose further that there exist $\alpha, \beta>0$ such that

$\left(D_{1}\right)$ For each $i=1,2, \ldots, n$, there exists a continuous function $\psi_{i}: N \rightarrow(0, \infty)$ such that

$$
f^{i}(j, u) \geq \phi\left(\frac{1}{T+1} \alpha\right) \psi_{i}(j), \quad \text { for all } j \in N, \quad \frac{\alpha}{T+1} \leq\langle v, u(j)\rangle \leq \alpha
$$

and

$$
\min _{k \in N}\langle v, P(k)\rangle \geq 1
$$

where $P(k)=\left(P_{1}(k), P_{2}(k), \ldots, P_{n}(k)\right)$ is the unique solution of

$$
\left\{\begin{array}{l}
\Delta(\phi(\Delta P(k-1)))+\psi(k)=0, \quad k \in \mathbb{N}, \\
P(0)=0, \quad P(T+1)=0
\end{array}\right.
$$

with $\psi=\left(\psi_{1}, \psi_{2}, \ldots, \psi_{n}\right)$.

$\left(\mathrm{D}_{2}\right)$ For each $i=1,2, \ldots, n$, there exists a continuous function $\chi_{i}: N \rightarrow(0, \infty)$ such that

$$
\left.f^{i}(j, u)\right\rangle \leq \phi(\beta) \chi_{i}(j), \quad \text { for all } j \in N, \quad 0<\langle v, u(j)\rangle \leq \beta
$$

and

$$
\max _{k \in N}|v, Q(k)\rangle \leq 1
$$

where $Q(k)=\left(Q_{1}(k), Q_{2}(k), \ldots, Q_{n}(k)\right)$ is the unique solution of

$$
\left\{\begin{array}{l}
\Delta(\phi(\Delta Q(k-1)))+\chi(k)=0, \quad k \in \mathbb{N}, \\
Q(0)=0, \quad Q(T+1)=0
\end{array}\right.
$$

with $\chi=\left(\chi_{1}, \chi_{2}, \ldots, \chi_{n}\right)$.

Then the following results hold:

(a) If $\beta<\frac{\alpha}{T+1}$, then system (1) has at least one positive solution $u$ with

$$
\beta \leq|u|_{v} \leq \alpha
$$

(b) If $\alpha<\beta$, then system (1) has at least one positive solution $u$ with

$$
\frac{\alpha}{T+1} \leq|u|_{v} \leq \beta
$$

Proof To obtain the desired result, we claim that

(i) $u \neq \lambda S u$ for $\lambda \in[0,1)$ and $u \in \partial_{K} B^{\beta}$, and

(ii) there exists $e \in K \backslash\{0\}$ such that $u \neq S u+\lambda e$ for each $u \in \partial_{K} \Omega^{\alpha}$ and all $\lambda>0$. 
We start with (i). Suppose that there exist $u \in \partial_{K} B^{\beta}$ and $\lambda \in[0,1)$ such that $u=\lambda S u$. Since $u \in \partial_{K} B^{\beta}$, we have $\frac{1}{T+1} \beta \leq\langle v, u(k)\rangle \leq \beta$ for all $k \in N$ and $\left\langle v, u\left(k^{*}\right)\right\rangle=\beta$ for some $k^{*} \in N$. From $\left(D_{2}\right)$ we get

$$
\Delta\left(\phi\left(\Delta S_{i} u(k-1)\right)\right)=-f^{i}(k, u(k)) \geq-\phi(\beta) \chi_{i}(k)=\Delta\left(\phi\left(\Delta \beta Q_{i}(k-1)\right)\right) .
$$

By Lemma 2.4, we obtain

$$
\beta=\left\langle v, u\left(k^{*}\right)\right\rangle=\left\langle v, \lambda \operatorname{Su}\left(k^{*}\right)\right\rangle \leq \lambda \beta\left\langle v, Q\left(k^{*}\right)\right\rangle \leq \lambda \beta \max _{k^{*} \in N}\left\langle v, Q\left(k^{*}\right)\right\rangle<\beta,
$$

which is a contradiction. Then (i) is proved.

Next we consider claim (ii). Let $e(t) \equiv 1$. Then $e \in K \backslash\{0\}$. Suppose, contrary to our claim, that there exist $u \in \partial_{K} \Omega^{\alpha}$ and $\lambda>0$ such that $u=S u+\lambda e$. By Lemma 3.2(d), for any $u \in \partial_{K} \Omega^{\alpha}$, we have $\frac{1}{T+1} \alpha \leq\langle v, u(k)\rangle \leq \alpha$ for $k \in N$.

It follows from $\left(D_{1}\right)$ that

$$
\Delta\left(\phi\left(\Delta S_{i} u(k-1)\right)\right)=-f^{i}(k, u(k)) \leq-\phi\left(\frac{\alpha}{T+1}\right) \psi_{i}(k)=\Delta\left(\phi\left(\Delta \frac{\alpha}{T+1} P_{i}(k-1)\right)\right) .
$$

We conclude from Lemma 2.4 that

$$
\begin{aligned}
\langle v, u(k)\rangle & =\langle v, \operatorname{Su}(k)+\lambda e\rangle=\langle v, \operatorname{Su}(k)\rangle+\langle v, \lambda e\rangle \\
& \geq \frac{\alpha}{T+1}\langle v, P(k)\rangle+\langle v, \lambda e\rangle \\
& \geq \frac{\alpha}{T+1} \min _{k \in N}\langle v, P(k)\rangle+\langle v, \lambda e\rangle>\frac{\alpha}{T+1},
\end{aligned}
$$

hence that $\min _{k \in N}\langle v, u(k)\rangle>\frac{\alpha}{T+1}$, and finally that this is a contradiction to Lemma 3.2(c). According to Lemma 3.2, if $\beta<\frac{\alpha}{T+1}$, one has $\bar{B}^{\beta}{ }_{K} \subset B_{K}^{\alpha / T+1} \subset \Omega_{K}^{\alpha}$. Now Theorem 2.5 guarantees the existence of at least one fixed point $u \in \overline{\Omega^{\alpha}}{ }_{K} \backslash B_{K}^{\beta}$ of $S$, so we have $|u|_{v} \geq$ $\beta$ and $\frac{1}{T+1} \beta \leq \min _{k \in N}\langle v, u(k)\rangle \leq \frac{1}{T+1} \alpha$. Moreover, $\frac{1}{T+1}|u|_{v} \leq \min _{k \in N}\langle v, u(k)\rangle \leq \frac{1}{T+1} \alpha$. We thus get $|u|_{v} \leq \alpha$.

On the other hand, if $\alpha<\beta$, one has ${\overline{\Omega^{\alpha}}}_{K} \subset B_{K}^{\beta}$. Similarly, we can obtain that $S$ has at least one fixed point $u \in \bar{B}^{\beta}{ }_{K} \backslash \Omega_{K}^{\alpha}$ by Theorem 2.5, which implies that $\frac{1}{T+1} \alpha \leq|u|_{\nu} \leq \beta$.

Remark 3.4 Take $p=2$ and the original system (1) is transformed into a common second order discrete boundary value system

$$
\left\{\begin{array}{l}
\Delta^{2} u_{i}(k-1)+f^{i}(k, u(k))=0, \quad k \in N, \\
u(0)=0, \quad u(T+1)=0, \quad i=1,2, \ldots, n
\end{array}\right.
$$

then system (9) has at least one positive solution.

\section{Eigenvalue intervals of (2)}

In this section, we employ Theorem 3.3 to establish one existence result for system (3), and then characterize the eigenvalue intervals of system (2). First we assume that 
$\left(\mathrm{H}_{1}\right) g^{i}: \mathbb{R}_{+}^{n} \rightarrow \mathbb{R}_{+}$is continuous with $g^{i}(u)>0$ for $|u|_{v}>0$;

$\left(\mathrm{H}_{2}\right) \widetilde{H}(k)>0$ for $k \in N$, where $\widetilde{H}(k)=\left(\widetilde{H}_{1}(k), \widetilde{H}_{2}(k), \ldots, \widetilde{H}_{n}(k)\right)$ is the unique solution of

$$
\left\{\begin{array}{l}
\Delta(\phi(\Delta u(k-1)))+h(k)=0, \quad k \in \mathbb{N}, \\
u(0)=0, \quad u(T+1)=0,
\end{array}\right.
$$

with $h(k)=\left(h_{1}(k), h_{2}(k), \ldots, h_{n}(k)\right)$.

Theorem 4.1 Assume that $\left(\mathrm{H}_{1}\right)$ and $\left(\mathrm{H}_{2}\right)$ hold. If one of the following conditions holds, then system (3) has at least one positive solution $u$ satisfying $\langle v, u(k)\rangle \neq 0$ for $k \in N$.

$\left(\mathrm{h}_{1}\right) 0 \leq g_{0}^{i}<\left(\frac{1}{M}\right)^{p-1}$ and $\left(\frac{1}{m}\right)^{p-1}<g_{\infty}^{i} \leq \infty, i=1,2, \ldots, n$;

$\left(\mathrm{h}_{2}\right) \quad 0 \leq g_{\infty}^{i}<\left(\frac{1}{M}\right)^{p-1}$ and $\left(\frac{1}{m}\right)^{p-1}<g_{0}^{i} \leq \infty, i=1,2, \ldots, n$;

where $g_{0}^{i}=\lim _{u \rightarrow 0^{+}} \frac{g^{i}(u)}{u^{p-1}}, g_{\infty}^{i}=\lim _{u \rightarrow \infty} \frac{g^{i}(u)}{u^{p-1}}$, and

$$
M=\max _{k \in N}\langle v, \widetilde{H}(k)\rangle, \quad m=\min _{k \in N}\langle v, \widetilde{H}(k)\rangle .
$$

Proof For this purpose, we set $f^{i}(k, u)=h_{i}(k) g^{i}(u), i=1,2, \ldots, n$, and suppose that $\left(\mathrm{h}_{1}\right)$ holds. In addition, the case when $\left(\mathrm{h}_{2}\right)$ holds is similar.

From the first part of $\left(\mathrm{h}_{1}\right)$, there exists $\beta>0$ such that $g^{i}(u) \leq\left(\frac{1}{M}\right)^{p-1} \beta^{p-1}$ for $0<|u|_{v} \leq \beta$. Take $\chi_{i}(k)=\left(\frac{1}{M}\right)^{p-1} h_{i}(k)$. When $0<\langle v, u(k)\rangle \leq \beta$, then

$$
f^{i}(k, u)=h_{i}(k) g^{i}(u) \leq\left(\frac{1}{M}\right)^{p-1} \beta^{p-1} h_{i}(k)=\beta^{p-1} \chi_{i}(k) \quad \text { for } k \in N
$$

and

$$
\langle v, Q(k)\rangle=\left\langle v, M^{-1} \widetilde{H}(k)\right\rangle \leq M^{-1} \max _{k \in N}\langle v, \widetilde{H}(k)\rangle=1
$$

Hence $\left(D_{2}\right)$ holds.

From the second part of $\left(\mathrm{h}_{1}\right)$, there exists $\alpha>0$ such that $\frac{1}{T+1} \alpha>\beta$ and $g^{i}(u) \geq$ $\left(\frac{1}{m}\right)^{p-1}\left(\frac{1}{T+1} \alpha\right)^{p-1}$ for $|u|_{v} \geq \frac{1}{T+1} \alpha$. Take $\psi_{i}(k)=\left(\frac{1}{m}\right)^{p-1} h_{i}(k)$. When $\frac{1}{T+1} \alpha \leq\langle v, u(k)\rangle \leq \alpha$, we have, for $k \in N$, that

$$
f^{i}(k, u)=h_{i}(k) g^{i}(u) \geq\left(\frac{1}{m}\right)^{p-1}\left(\frac{1}{T+1} \alpha\right)^{p-1} h_{i}(k)=\left(\frac{1}{T+1} \alpha\right)^{p-1} \psi_{i}(k)
$$

and

$$
\langle v, P(k)\rangle=\left\langle v, m^{-1} \widetilde{H}(k)\right\rangle \geq m^{-1} \min _{k \in N}\langle v, \widetilde{H}(k)\rangle=1
$$

This implies that $\left(D_{1}\right)$ holds. Now the rest of the proof runs as Theorem 3.3.

Next we employ Theorem 4.1 to characterize the eigenvalue intervals for system (2). As it is easy to prove, we only present the results here. 
Theorem 4.2 Assume that $\left(\mathrm{H}_{1}\right)$ and $\left(\mathrm{H}_{2}\right)$ hold. If $\frac{1}{m^{p-1} \min _{i=1,2, \ldots, n}\left\{g_{\infty}^{i}\right\}}<\frac{1}{M^{p-1} \max _{i=1,2, \ldots, n}\left\{g_{0}^{i}\right\}}$, then system (2) has at least one positive solution for each

$$
\begin{gathered}
\lambda \in\left(\frac{1}{m^{p-1} \min _{i=1,2, \ldots, n}\left\{g_{\infty}^{i}\right\}}, \frac{1}{M^{p-1} \max _{i=1,2, \ldots, n}\left\{g_{0}^{i}\right\}}\right) . \\
\text { If } \frac{1}{m^{p-1} \min _{i=1,2, \ldots, n}\left\{g_{0}^{i}\right\}}<\frac{1}{M^{p-1} \max _{i=1,2, \ldots, n}\left\{g_{\infty}^{i}\right\}}, \text { the same result remains valid for each } \\
\lambda \in\left(\frac{1}{m^{p-1} \min _{i=1,2, \ldots, n}\left\{g_{0}^{i}\right\}}, \frac{1}{M^{p-1} \max _{i=1,2, \ldots, n}\left\{g_{\infty}^{i}\right\}}\right) .
\end{gathered}
$$

\section{Conclusions}

In this paper, we established the existence of positive solutions for the second order $p$ Laplacian discrete system by a well-known fixed point theorem in cones. In this paper, we will choose another adequate cone to prove the main results, which will improve and generalize those results obtained in [6]. Moreover, we take advantage of a new cone to characterize the eigenvalue intervals in a simple way.

\section{Acknowledgements}

The author is grateful to anonymous referees for their constructive comments and suggestions which have greatly improved this paper. At the same time, the author would like to thank Professor Chu Jifeng for his valuable comments.

\section{Funding}

This research is partially supported by the National Natural Science Foundation of China (Grant No. 11671118).

Availability of data and materials

Not applicable.

\section{Competing interests}

The author declares that there is no conflict of interests regarding the publication of this paper.

\section{Authors' contributions}

The author read and approved the final manuscript.

\section{Publisher's Note}

Springer Nature remains neutral with regard to jurisdictional claims in published maps and institutional affiliations.

Received: 24 April 2018 Accepted: 6 August 2018 Published online: 14 August 2018

\section{References}

1. Agarwal, R.P., O'Regan, D.: Nonpositive discrete boundary value problems. Nonlinear Anal. 39, 207-215 (2000)

2. Agarwal, R.P., O'Regan, D., Wong, P.J.Y.: Positive Solutions of Differential, Difference and Integral Equations. Kluwer Academic, Dordrecht (1999)

3. Agarwal, R.P., Perera, K., O'Regan, D.: Multiple positive solutions of singular discrete $p$-Laplacian problems via variational methods. Adv. Differ. Equ. 2, 93-99 (2005)

4. Avery, R., Henderson, J.: Existence of three positive pseudo-symmetric solutions for a one dimensional discrete p-Laplacian. J. Differ. Equ. Appl. 10, 529-539 (2004)

5. Chu, J., Jiang, D.: Eigenvalues and discrete boundary value problems for the one-dimensional $p$-Laplacian. J. Math. Anal. Appl. 305, 452-465 (2005)

6. Chu, J., O'Regan, D.: Positive solutions and eigenvalue intervals for discrete boundary value problems. Commun. Appl. Anal. 12, 245-264 (2008)

7. Heidarkhani, S., Caristi, G., Salari, A.: Perturbed Kirchhoff-type $p$-Laplacian discrete problems. Collect. Math. 68, 401-418 (2017)

8. Henderson, J., Luca, R.: Existence of positive solutions for a system of second-order multi-point discrete boundary value problems. J. Differ. Equ. Appl. 19, 1889-1906 (2013)

9. Jiang, D., Chu, J., O'Regan, D., Agarwal, R.P.: Positive solutions for continuous and discrete boundary value problems to the one-dimension p-Laplacian. Math. Inequal. Appl. 4, 523-534 (2004)

10. Jiang, D., O'Regan, D., Agarwal, R.P.: A generalized upper and lower solution method for singular discrete boundary value problems for the one-dimensional p-Laplacian. J. Appl. Anal. 11, 35-47 (2005)

11. Jiang, D., Pang, P.Y.H., Agarwal, R.P.: Upper and lower solutions method and a superlinear singular discrete boundary value problem. Dyn. Syst. Appl. 16, 743-753 (2007) 
12. King, S., Neugebauer, J.: Smallest eigenvalues, extremal points, and positive solutions of a fourth order three point boundary value problem. Dyn. Syst. Appl. 23, 699-713 (2014)

13. Krasnosel'skii, M.A.: Positive Solutions of Operator Equations. Noordhoff, Groningen (1964)

14. Krushna, B.M.B., Prasad, K.R.: Eigenvalue intervals for the existence of positive solutions to system of multi-point fractional order boundary value problems. J. Int. Math. Virtual Inst. 6, 49-65 (2016)

15. Nastasi, A., Vetro, C., Vetro, F.: Positive solutions of discrete boundary value problems with the $(p, q)$-Laplacian operator. Electron. J. Differ. Equ. 2017, 225 (2017)

16. Tang, $X$. : Existence and uniqueness of nontrivial solutions for eigenvalue boundary value problem of nonlinear fractional differential equation. Ann. Univ. Ferrara, Sez. 7: Sci. Mat. 60, 429-445 (2014)

17. Tian, Y., Ge, W.: Existence of multiple positive solutions for discrete problems with $p$-Laplacian via variational methods. Electron. J. Differ. Equ. 2011, 45 (2011)

18. Yao, Q.: Positive solutions and eigenvalue intervals of a nonlinear singular fourth-order boundary value problem. Appl. Math. 58, 93-110 (2013)

19. Yuan, C., Jiang, D., O'Regan, D.: Existence and uniqueness of positive solutions for fourth-order nonlinear singular continuous and discrete one-dimension p-Laplacian problems. Panam. Math. J. 18, 31-46 (2008)

Submit your manuscript to a SpringerOpen ${ }^{\circ}$ journal and benefit from:

- Convenient online submission

- Rigorous peer review

Open access: articles freely available online

High visibility within the field

Retaining the copyright to your article

Submit your next manuscript at $\boldsymbol{~ s p r i n g e r o p e n . c o m ~}$ 